\title{
Accelerated nonstandard finite difference method for singularly perturbed Burger-Huxley equations
}

\author{
Masho Jima Kabeto* and Gemechis File Duressa
}

\begin{abstract}
Objective: The main purpose of this paper is to present an accelerated nonstandard finite difference method for solving the singularly perturbed Burger-Huxley equation in order to produce more accurate solutions.

Results: The quasilinearization technique is used to linearize the nonlinear term. A nonstandard methodology of Mickens procedure is used in the spatial direction and also within the first order temporal direction that construct the first-order finite difference approximation to solve the considered problem numerically. To accelerate the rate of convergence from first to second-order, the Richardson extrapolation technique is applied. Numerical experiments were conducted to support the theoretical results.
\end{abstract}

Keywords: Singularly perturbed Burger-Huxley equation, Accelerated nonstandard method, Accurate solution Mathematics Subject Classification: Primary 65M06, 65M12, Secondary 65M15

\section{Introduction}

Singularly perturbed differential equations are typically characterized by a small perturbation parameter multiplied with the highest order derivative term. These types of equations appear in computational fluid dynamics, hydrodynamics, chemical reactor theory, financial modeling, mathematical biology [1-3]. The solutions of singularly perturbed problems exhibit layer. The conventional numerical methods on uniform meshes do not produce satisfactory numerical approximations for small values of the perturbation parameter. A uniformly convergent numerical method, which is a numerical method suitable for these problems and in which the error bound is independent of the size of the perturbation parameter (see [4, 5] and the references therein for more details).

In this work the singularly perturbed Burger-Huxley equations of the form:

$$
\left\{\begin{array}{l}
L_{x, \varepsilon} u(x, t) \cong-\varepsilon \frac{\partial^{2} u}{\partial x^{2}}+\alpha u \frac{\partial u}{\partial x}+\frac{\partial u}{\partial t}-\beta(1-u)(u-\gamma) u=0, \quad \forall(x, t) \in D \\
u(x, 0)=u_{0}(x), \quad x \in[0,1], \quad u(0, t)=s_{0}(t), \quad u(1, t)=s_{1}(t), \quad t \in(0, T]
\end{array}\right.
$$

\footnotetext{
*Correspondence: maashookoo.reemii@gmail.com

Department of Mathematics, Jimma University, Jimma, Ethiopia
} 
where $0<\varepsilon<<1$ is a perturbation parameter. The solution domain $D=(0,1) \times(0, T]$, and $\alpha \geq 1, \beta \geq 0$, $\gamma \in(0,1)$ are given constants.

Burger-Huxley equation describes the interaction between convection, diffusion, and reaction processes that have numerous fascinating phenomena such as busting oscillation, population genetics, bifurcation, and so on [6-11]. A numerical method for Eq. (1) has been developed only in [6] using a robust adaptive grid method, a uniformly convergent method, and a singular perturbation approach respectively. However, all these methods considered the standard backward Euler scheme. To solve Eq. (1) the idea of using classical numerical methods is not an efficient approach to produce an accurate solution. This should be develop an accelerated nonstandard finite difference method. As a general rule, higher-order convergent methods are preferred as they provide well numerical approximations with low computational cost. So, in this paper, our objective is to propose the accelerated nonstandard finite difference method for solving Eq. (1) by applying the nonstandard procedures of Mickens [12] in the space direction. We provide an error analysis for the method and prove that it is uniformly convergent with second-order accuracy after applying the Richardson extrapolation technique. It is also shown that the method is computationally more efficient compared to some existing methods in the literature.

\section{Main text}

\section{Numerical method}

Consider equation under consideration in Eq. (1), rewritten as:

$$
\left\{\begin{array}{l}
\frac{\partial u}{\partial t}-\varepsilon \frac{\partial^{2} u}{\partial x^{2}}=F\left(x, t, u, \frac{\partial u}{\partial x}\right), \quad \forall(x, t) \in D, \\
u(x, 0)=u_{0}(x), \quad x \in[0,1], \quad u(0, t)=s_{0}(t), \quad u(1, t)=s_{1}(t), \quad t \in(0, T],
\end{array}\right.
$$

guess intial approximation in the linearization process. Thus, to linearize Eq. (2), by applying the quasilinearization technique on the nonlinear term, for the reasonable initial guess of the form of Eq. (4) is given by:

$$
u^{(0)}(x, t)=u_{0}(x) \exp \left(-\pi^{2} t\right) .
$$

Thus, the nonlinear term $F\left(x, t, u, \frac{\partial u}{\partial x}\right)$ can be linearized initially as:

$$
\begin{aligned}
& F\left(x, t, u^{(1)}, \frac{\partial u^{(1)}}{\partial x}\right) \cong F\left(x, t, u^{(0)}, \frac{\partial u^{(0)}}{\partial x}\right) \\
& +\left.\left(u^{(1)}-u^{(0)}\right) \frac{\partial F}{\partial u}\right|_{u^{(0)}} \\
& \quad+\left.\left(\frac{\partial u^{(1)}}{\partial x}-\frac{\partial u^{(0)}}{\partial x}\right) \frac{\partial F}{\partial\left(\frac{\partial u}{\partial x}\right)}\right|_{\frac{\partial u^{(0)}}{\partial x}} .
\end{aligned}
$$

Substituting Eq. (6) into Eq. (2) and inducing for iteration number $i$, we obtain the linearized differential equation.

$$
\begin{aligned}
& \frac{\partial u^{(i+1)}}{\partial t}-\varepsilon \frac{\partial^{2} u^{(i+1)}}{\partial x^{2}}+a^{(i)}(x, t) \frac{\partial u^{(i+1)}}{\partial x} \\
& +b^{(i)}(x, t) u^{(i+1)}=f^{(i)}(x, t), \\
& \quad a^{(i)}(x, t)=-\left.\frac{\partial F}{\partial\left(\frac{\partial u}{\partial x}\right)}\right|_{\left(x, t, u^{(i)}, \frac{\partial u^{(i)}}{\partial x}\right)}, \ldots b^{(i)}(x, t)
\end{aligned}
$$

$$
=-\left.\frac{\partial F}{\partial u}\right|_{\left(x, t, u^{(i)}, \frac{\partial u^{(i)}}{\partial x}\right)^{\prime}}
$$

where

where $F\left(x, t, u, \frac{\partial u}{\partial x}\right)=\beta(1-u)(u-\gamma) u-\alpha u \frac{\partial u}{\partial x}$.

Let us consider the homogenous part of Eq. (2):

$$
\left\{\begin{array}{l}
\frac{\partial u}{\partial t}-\varepsilon \frac{\partial^{2} u}{\partial x^{2}}=0 \quad \forall(x, t) \in D, \\
u(x, 0)=u_{0}(x), \quad x \in[0,1], \quad u(0, t)=s_{0}(t), u(1, t)=s_{1}(t), \quad t \in(0, T] .
\end{array}\right.
$$

We look for a solution to the dimensionless heat equation of the form of Eq. (3), when $\varepsilon=1$, using separation of variables, we get the solution to Eq. (3) as:

$$
u(x, t)=u_{0}(x) \exp \left(-\pi^{2} t\right),
$$

that satisfies both the initial and boundary conditions given in Eq. (2). The formula in Eq. (4) will be used to 
partitioned into $N$ equal sub-intervals with each nodal point satisfies: $0=t_{0}<t_{1}<\cdots<t_{N}=T$. Thus, the temporal nodal points are generated by

$$
t_{n}=n k, \quad k=\frac{T}{N}, \quad n=0,1, \ldots, N
$$

Using Taylor's series expansion, we have:

$u^{n+1}(x)=u^{n}(x)+k \frac{\partial u^{n}(x)}{\partial t}+\frac{k^{2}}{2} \frac{\partial^{2} u^{n}(x)}{\partial t^{2}}+\frac{k^{3}}{6} \frac{\partial^{3} u^{n}(x)}{\partial t^{3}}+\cdots$, which gives:

$$
\frac{\partial u^{n}}{\partial t} \cong \frac{u^{n+1}(x)-u^{n}(x)}{k}+T E,
$$

where the truncation term is $T E=-\frac{k}{2} \frac{\partial^{2} u^{n}(x)}{\partial t^{2}}$. This indi-
cates, the error estimate:

$$
\|E\|_{\infty} \leq C k,
$$

for the arbitrary constant $C=\frac{1}{2}\left\|\frac{\partial^{2} u^{n}(x)}{\partial t^{2}}\right\|_{\infty}$. Substituting Eq. (9) into Eq. (7) gives the semi-discrete differential equation of the form:
Substituting the values of $\lambda_{1}$ and $\lambda_{2}$ into Eq. (13), we get:

$$
\begin{aligned}
u_{m-1} \exp \left(\frac{-\eta h}{2 \varepsilon}\right) & -2 \cosh \left(\frac{h \sqrt{\eta^{2}+4 \varepsilon \theta}}{2 \varepsilon}\right) \\
& +u_{m+1} \exp \left(\frac{\eta h}{2 \varepsilon}\right)=0 .
\end{aligned}
$$

This Eq. (15) is the exact difference scheme of Eq. (12), in the wisdom that the difference Eq. (15) has the general solution $u_{m}=C_{1} \exp \left(\lambda_{1} x_{m}\right)+C_{2} \exp \left(\lambda_{2} x_{m}\right)$ as the differential Eq. (12). Since, $\theta \geq 0$, we require the exact scheme for the reduced case. To this end, the exact scheme corresponding to $\varepsilon \frac{d^{2} u(x)}{d x^{2}}+\eta \frac{d u(x)}{d x}=0$. Thus, Eq. (15) is deduced to:

$$
\begin{aligned}
u_{m-1} \exp \left(\frac{-\eta h}{2 \varepsilon}\right) & -2 \cosh \left(\frac{\eta h}{2 \varepsilon}\right) u_{m} \\
& +u_{m+1} \exp \left(\frac{\eta h}{2 \varepsilon}\right)=0 .
\end{aligned}
$$

$$
\left\{\begin{array}{l}
\left(-\varepsilon \frac{\partial^{2} u^{(i+1)}}{\partial x^{2}}+a^{(i)} \frac{\partial u^{(i+1)}}{\partial x}+\left(b^{(i)}+\frac{1}{k}\right) u^{(i+1)}\right)\left(x, t_{n+1}\right)=\left(f^{(i)}+\frac{1}{k} u^{(i)}\right)\left(x, t_{n+1}\right), \\
u(x, 0)=u_{0}(x), \quad x \in[0,1], \quad u\left(0, t_{n+1}\right)=s_{0}\left(t_{n+1}\right), \quad u\left(1, t_{n+1}\right)=s_{1}\left(t_{n+1}\right) .
\end{array}\right.
$$

Let us consider the singularly perturbed convectiondiffusion differential equation of the form:

$$
\varepsilon \frac{d^{2} u(x)}{d x^{2}}+\eta \frac{d u(x)}{d x}-\theta u(x)=0, \quad x \in(0,1),
$$

where $\eta>0$ and $\theta \geq 0$ are constants. Then, Eq. (12) has two linearly independent solutions as:

$$
u_{1}(x)=\exp \left(\lambda_{1} x\right), \quad \text { and } \quad u_{2}(x)=\exp \left(\lambda_{2} x\right)
$$

for the constants $\lambda_{1}=\frac{-\eta+\sqrt{\eta^{2}+4 \varepsilon \theta}}{2 \varepsilon}$, and $\lambda_{2}=$ $\frac{-\eta-\sqrt{\eta^{2}+4 \varepsilon \theta}}{2 \varepsilon}$. Let $M$ be is a positive integer, and the interval $[0,1]$ on the spatial direction be divided into $M$ equal sub-intervals through the nodes $x_{m}=m h, \quad m=\frac{1}{M}, \quad m=0,1, \ldots, M, \quad$ and the approximate solution to $u(x)$ at the grid points $x_{m}^{\prime} s$ is denoted by $u_{m}^{\prime} s$. The theory of difference (see [12]), shows that the second-order linear difference equation:

$$
\begin{aligned}
& u_{m-1}\left(\exp \left(\lambda_{2} h\right)-\exp \left(\lambda_{1} h\right)\right) \\
& \quad-u_{m}\left(\exp \left(\lambda_{2}-\lambda_{1}\right) h-\exp \left(\lambda_{1}-\lambda_{2}\right) h\right) \\
& \quad+u_{m+1}\left(\exp \left(-\lambda_{1} h\right)-\exp \left(\lambda_{2} h\right)\right)=0 .
\end{aligned}
$$

Multiplying both sides of Eq. (16) by exp $\left(\frac{\eta h}{2 \varepsilon}\right)$, and incorporating the term $u_{m+1}-u_{m}$ into this equation, we get:

$$
u_{m-1}-2 u_{m}+u_{m+1}+\left(u_{m+1}-u_{m}\right)\left(\exp \left(\frac{\eta h}{\varepsilon}\right)-1\right)=0 .
$$

Consequently, Eq. (17) can be transformed into:

$$
\varepsilon \frac{u_{m-1}-2 u_{m}+u_{m+1}}{\phi^{2}}+\eta \frac{u_{m+1}-u_{m}}{h}=0,
$$

where $\phi^{2}=\frac{h \varepsilon}{\eta}\left(\exp \left(\frac{\eta h}{\varepsilon}\right)-1\right)$.

When we come to the Burger-Huxley equation, the differential equation under consideration the full discretization of $\bar{D}_{M}^{N}$, denoting to approximation $u\left(x_{m}, t_{n}\right)$ by $U_{m}^{n}$. Then, the nonstandard finite difference rules developed for ODE above can be extended for PDE is given by:

$$
\left\{\begin{aligned}
L_{M}^{N} U \cong- & \varepsilon\left(\frac{U_{m-1}^{n+1}-2 U_{m}^{n+1}+U_{m+1}^{n+1}}{\phi_{m}^{2}}\right)^{i+1}+\left(a_{m}^{n+1}\right)^{i+1}\left(\frac{U_{m}^{n+1}-U_{m-1}^{n+1}}{h}\right)^{i+1} \\
& +\left(\left(b_{m}^{n+1}\right)^{i+1}+\frac{1}{k}\right)\left(U_{m}^{n+1}\right)^{i+1}=\left(f_{m}^{n+1}\right)^{i+1}+\frac{1}{k}\left(U_{m}^{n}\right)^{i+1} \\
U_{m}^{0}= & u_{0}\left(x_{m}\right), \quad U_{0}^{n+1}=s_{0}\left(t_{n+1}\right), \quad U_{M}^{n+1}=s_{1}\left(t_{n+1}\right)
\end{aligned}\right.
$$


where $i=0,1, \ldots \quad$ iteration number, and $\phi_{m}^{2}=\frac{h \varepsilon}{a_{m}^{n+1}}\left(\exp \left(\frac{h a_{m}^{n+1}}{\varepsilon}\right)-1\right)$.

\section{Stability of the scheme}

A partial differential equation is well-posed if the solution of the partial differential equation is exists, and depends continuously on the initial condition and boundary conditions. The Von Neumann stability technique is applied to investigate the stability of the developed scheme in Eq. (19), by assuming that the solution of Eq. (19) at the grid point $\left(x_{m}, t_{n}\right)$ is given by:

$$
U_{m}^{n}=\xi^{n} e^{i m \theta} .
$$

where $i=\sqrt{-1}, \quad \theta$ is the real number and $\xi$ is the amplitude factor.

Now, considering at the first iteration and putting Eq. (20) into the homogeneous scheme part of Eq. (19) gives:

$$
\begin{aligned}
& \frac{-\varepsilon}{\phi_{m}^{2}}\left(\xi^{n+1} e^{i(m-1) \theta}-2 \xi^{n+1} e^{i m \theta}+\xi^{n+1} e^{i(m+1) \theta}\right) \\
& +\frac{a_{m}^{n+1}}{h}\left(\xi^{n+1} e^{i m \theta}-\xi^{n+1} e^{i(m-1) \theta}\right) \\
& +b_{m}^{n+1} \xi^{n+1} e^{i m \theta}+\frac{1}{k}\left(\xi^{n+1} e^{i m \theta}-\xi^{n} e^{i m \theta}\right)=0 .
\end{aligned}
$$

Then, solving for the amplitude factor $\xi$, yields:

$$
\begin{aligned}
\xi & =\frac{\frac{1}{k}}{\frac{-\varepsilon}{\phi_{m}^{2}}\left(e^{-i \theta}-2+e^{i \theta}\right)+\frac{a_{m}^{n+1}}{h}\left(1-e^{-i \theta}\right)+b_{m}^{n+1}+\frac{1}{k}} \\
& =\frac{1}{1-\frac{-\varepsilon k}{\phi_{m}^{2}}\left(e^{-i \theta}-2+e^{i \theta}\right)+\frac{k a_{m}^{n+1}}{h}\left(1-e^{-i \theta}\right)+k b_{m}^{n+1}} .
\end{aligned}
$$

The condition of stability is $\xi \leq 1$ and for sufficiently small $k$, we have $\xi=1$. Hence, the scheme given in Eq. (19) is stable. Thus, the scheme in Eq. (19) is unconditionally stable.

\section{Consistency of the scheme}

The local truncation error $T(h, k)$ between the operator on the exact solution $u_{m}^{n}$ to Eq. (7) and the approximate solution $U_{m}^{n}$ to Eq. (19) at the fixed $i=0$ iteration is given by:

$$
\begin{aligned}
T(h, k)=\frac{\partial u_{m}^{n+1}}{\partial t}-\varepsilon \frac{\partial^{2} u_{m}^{n+1}}{\partial x^{2}}+a_{m}^{n+1} \frac{\partial u_{m}^{n+1}}{\partial x}+b_{m}^{n+1} u_{m}^{n+1} \\
-\left(\frac{U_{m}^{n+1}-U_{m}^{n}}{k}-\varepsilon \frac{U_{m+1}^{n+1}-2 U_{m}^{n+1}+U_{m-1}^{n+1}}{\phi_{m}^{2}}\right. \\
\left.+a_{m}^{n+1} \frac{U_{m}^{n+1}-U_{m-1}^{n+1}}{h}+b_{m}^{n+1} U_{m}^{n+1}\right) .
\end{aligned}
$$

Using Taylor's series expansion, we have:

$$
\begin{aligned}
& \frac{U_{m}^{n+1}-U_{m}^{n}}{k}=\frac{\partial u_{m}^{n+1}}{\partial t}+\frac{k}{2} \frac{\partial^{2} u_{m}^{n+1}}{\partial t^{2}}+O\left(k^{2}\right) . \\
& U_{m+1}^{n+1}-2 U_{m}^{n+1}+U_{m-1}^{n+1}=h^{2} \frac{\partial^{2} u_{m}^{n+1}}{\partial x^{2}}+O\left(h^{4}\right) . \\
& \frac{U_{m}^{n+1}-U_{m-1}^{n+1}}{h}=\frac{\partial u_{m}^{n+1}}{\partial x}+\frac{h}{2} \frac{\partial^{2} u_{m}^{n+1}}{\partial x^{2}}+O\left(h^{2}\right) .
\end{aligned}
$$

Substituting Eqs. (22)-(24) into Eq. (21), which implies:

$$
\begin{aligned}
T(h, k)= & -\frac{k}{2} \frac{\partial^{2} u_{m}^{n+1}}{\partial t^{2}}-\frac{h}{2} a_{m}^{n+1} \frac{\partial^{2} u_{m}^{n+1}}{\partial x^{2}} \\
& -\left(1-\frac{h^{2}}{\phi_{m}^{2}}\right) \varepsilon \frac{\partial^{2} u_{m}^{n+1}}{\partial x^{2}}+O\left(h^{2}+k^{2}\right) .
\end{aligned}
$$

Now, the values of $\phi_{m}^{2}$ provided in Eq. (19) can be expanded as:

$$
\phi_{m}^{2}=h^{2}+\frac{h^{3}}{2 !} \frac{a_{m}^{n+1}}{\varepsilon}+\frac{h^{4}}{3 !}\left(\frac{a_{m}^{n+1}}{\varepsilon}\right)^{2}+\frac{h^{5}}{4 !}\left(\frac{a_{m}^{n+1}}{\varepsilon}\right)^{3}+\cdots .
$$

Thus, the truncation error is

$$
T(h, k)=-\frac{k}{2} \frac{\partial^{2} u_{m}^{n+1}}{\partial t^{2}}-\frac{h}{2} a_{m}^{n+1} \frac{\partial^{2} u_{m}^{n+1}}{\partial x^{2}}+O\left(h^{2}+k^{2}\right) .
$$

Therefore, the norm of the local truncation error can be written as:

$$
|T(h, k)|=\left|L\left(u_{m}^{n+1}-U_{m}^{n+1}\right)\right| \leq C_{1} h+C_{2} k,
$$

where $C_{1}=\frac{1}{2}\left\|\frac{\partial^{2} u_{m}^{n+1}}{\partial t^{2}}\right\|_{\infty}$, and $C_{2}=\frac{1}{2}\left\|a_{m}^{n+1} \frac{\partial^{2} u_{m}^{n+1}}{\partial x^{2}}\right\|_{\infty}$.

To accelerate the rate of convergence, we apply the Richardson extrapolation techniques on Eq. (27). Assume that $U(h, k)$ denote the approximate value of $u\left(x_{m}, t_{n}\right)$ with the mesh length of $h$ and $k$. The approximate solution $U\left(\frac{h}{2}, \frac{k}{2}\right)$ also denotes the value of $u\left(x_{m}, t_{n}\right)$ obtained by using the same method with step length $\frac{h}{2}$ and $\frac{k}{2}$, then the order of convergence for the two approximate solutions can be written as:

$$
\left\{\begin{array}{l}
u\left(x_{m}, t_{n}\right)-U(h, k) \equiv C(h+k)+O\left(h^{2}+k^{2}\right), \\
u\left(x_{m}, t_{n}\right)-U\left(\frac{h}{2}, \frac{k}{2}\right) \equiv C\left(\frac{h}{2}+\frac{k}{2}\right)+O\left(h^{2}+k^{2}\right),
\end{array}\right.
$$

where $C$ is a constant independent of the perturbation and mesh parameters.

Eliminating C from Eq. (28), gives: 


$$
u\left(x_{m}, t_{n}\right)-2 U\left(\frac{h}{2}, \frac{k}{2}\right)+U(h, k) \equiv O\left(h^{2}+k^{2}\right) .
$$

Let us denote the combination of the two approximate solutions in Eq. (29) by:

$$
U_{(h, k)}^{e x t}=2 U\left(\frac{h}{2}, \frac{k}{2}\right)+U(h, k) .
$$

Then, it is re-written as:

$$
u\left(x_{m}, t_{n}\right)-U_{(h, k)}^{e x t}=O\left(h^{2}+k^{2}\right) .
$$

This indicates the method is accelerated to secondorder. Hence, we have

$$
\left|u\left(x_{m}, t_{n}\right)-U_{(h, k)}^{e x t}\right| \leq C\left(h^{2}+k^{2}\right) .
$$

Therefore, the proposed method is second-order uniformly convergent. Thus, the right hand side hand

$$
\begin{aligned}
E_{M}^{N} & =\max _{\forall\left(x_{m}, t_{n}\right) \in \bar{D}}\left|U_{m}^{n}-U_{2 m}^{2 n}\right|, \quad \text { and }\left(E_{M}^{N}\right)^{e x t} \\
& =\max _{\forall\left(x_{m}, t_{n}\right) \in \bar{D}}\left|\left(U_{m}^{n}\right)^{e x t}-\left(U_{2 m}^{2 n}\right)^{e x t}\right|,
\end{aligned}
$$

where $U_{m}^{n}$ and $U_{2 m}^{2 n}$ are approximate solutions evaluated on $\bar{D}_{M}^{N}$ and $\bar{D}_{2 M}^{2 N}$ respectively. Similarly, its extrapolated are induced. The corresponding rate of convergences is determined by:

$$
\begin{aligned}
R_{M}^{N} & =\frac{\log \left(E_{M}^{N}\right)-\log \left(E_{2 M}^{2 N}\right)}{\log (2)}, \text { and }\left(R_{M}^{N}\right)^{\text {ext }} \\
& =\frac{\log \left(\left(E_{M}^{N}\right)^{e t x}\right)-\log \left(\left(E_{2 M}^{2 N}\right)^{e x t}\right)}{\log (2)} .
\end{aligned}
$$

Example 1. Consider the singularly perturbed BurgersHuxley equation:

$$
\left\{\begin{array}{l}
\left(\frac{\partial u}{\partial t}-\varepsilon \frac{\partial^{2} u}{\partial x^{2}}+u \frac{\partial u}{\partial x}-(1-u)(u-0.5) u\right)(x, t)=0, \quad(x, t) \in(0,1) \times(0,1] \\
u(x, 0)=x\left(1-x^{2}\right), \quad 0<x<1, \quad u(0, t)=u(1, t)=0, \quad t \in[0,1] .
\end{array}\right.
$$

of Eq. (27) vanishes as $k \rightarrow 0$ and $h \rightarrow 0$ implies $T(h, k) \rightarrow 0$. Hence, the scheme is consistent with the order of convergence $O\left(k^{2}+h^{2}\right)$. Ended, the scheme
Example 2. Consider the following singularly perturbed Burgers' equation:

$$
\begin{cases}\left(\frac{\partial u}{\partial t}-\varepsilon \frac{\partial^{2} u}{\partial x^{2}}+u \frac{\partial u}{\partial x}\right)(x, t)=0, \quad(x, t) \in(0,1) \times(0,1], & \\ u(x, 0)=x\left(1-x^{2}\right), \quad 0<x<1, \quad u(0, t)=u(1, t)=0, \quad t \in[0,1] .\end{cases}
$$

developed in Eq. (19), is convergent. A consistent and stable finite difference method is convergent by Lax's equivalence theorem [2]. For further convergence analysis, one can see the works provided in $[5,11,13,14]$.

\section{Numerical illustration and discussions}

The exact solution for the considered examples is not available. Hence, the maximum absolute errors are calculated by the double mesh principle, [6], for before and after applying the Richardson extrapolation technique respectively by:
Tables 1 and 2 show the maximum absolute errors that demonstrate the validity of the present method and errors are monotonically decreasing behavior with increasing the number of intervals which confirm the convergence of the method. Table 3 validate that the corresponding rate of convergence. Thus, the proposed method is second-order convergent. Furthermore, the method gives a more accurate solution than some existing methods in the literature. 
Table 1 Comparison of maximum absolute errors for Example 1

\begin{tabular}{|c|c|c|c|c|c|}
\hline$\varepsilon \downarrow M / N \rightarrow$ & $32 / 20$ & $64 / 40$ & $128 / 80$ & $256 / 160$ & $512 / 320$ \\
\hline \multicolumn{6}{|c|}{ After extrapolation } \\
\hline $2^{-6}$ & $1.1939 \mathrm{e}-04$ & $3.4613 e-05$ & $9.3412 e-06$ & $4.2951 \mathrm{e}-06$ & $2.3138 \mathrm{e}-06$ \\
\hline $2^{-8}$ & $1.7054 \mathrm{e}-04$ & $5.6853 \mathrm{e}-05$ & $1.5929 \mathrm{e}-05$ & $4.2117 \mathrm{e}-06$ & $1.0822 \mathrm{e}-06$ \\
\hline $2^{-10}$ & $2.1124 e-04$ & $1.0056 \mathrm{e}-04$ & $3.8345 \mathrm{e}-05$ & $1.1640 \mathrm{e}-05$ & $3.1477 \mathrm{e}-06$ \\
\hline $2^{-12}$ & $2.0194 \mathrm{e}-04$ & $7.9291 \mathrm{e}-05$ & $5.5162 \mathrm{e}-05$ & $3.0062 \mathrm{e}-05$ & $1.0964 \mathrm{e}-05$ \\
\hline $2^{-18}$ & $2.7271 e-04$ & $7.2971 \mathrm{e}-05$ & $1.8774 \mathrm{e}-05$ & $4.6058 \mathrm{e}-06$ & $1.8644 \mathrm{e}-06$ \\
\hline \multicolumn{6}{|c|}{ Before extrapolation } \\
\hline $2^{-6}$ & $3.3878 \mathrm{e}-03$ & $1.7453 \mathrm{e}-03$ & $8.8211 \mathrm{e}-04$ & $4.4321 \mathrm{e}-04$ & $2.2212 \mathrm{e}-04$ \\
\hline $2^{-8}$ & $4.0171 \mathrm{e}-03$ & $2.0163 \mathrm{e}-03$ & $1.0020 \mathrm{e}-03$ & $4.9855 e-04$ & $2.4852 \mathrm{e}-04$ \\
\hline $2^{-10}$ & $4.4610 \mathrm{e}-03$ & $2.2241 \mathrm{e}-03$ & $1.0800 \mathrm{e}-03$ & $5.2600 \mathrm{e}-04$ & $2.5871 \mathrm{e}-04$ \\
\hline $2^{-12}$ & $4.6996 \mathrm{e}-03$ & $2.4258 \mathrm{e}-03$ & $1.2014 \mathrm{e}-03$ & $5.7660 e-04$ & $2.7486 e-04$ \\
\hline $2^{-18}$ & $4.7584 \mathrm{e}-03$ & $2.5003 e-03$ & $1.2810 \mathrm{e}-03$ & $6.4852 \mathrm{e}-04$ & $3.2604 \mathrm{e}-04$ \\
\hline \multicolumn{6}{|l|}{ Results in [6] } \\
\hline $2^{-6}$ & $2.5289 \mathrm{e}-02$ & $1.7672 \mathrm{e}-02$ & $9.0066 \mathrm{e}-03$ & $4.8378 \mathrm{e}-03$ & $2.5035 \mathrm{e}-03$ \\
\hline $2^{-8}$ & $3.8607 \mathrm{e}-02$ & $1.9497 \mathrm{e}-02$ & $1.1221 \mathrm{e}-02$ & $6.2852 \mathrm{e}-03$ & $3.3405 e-03$ \\
\hline $2^{-10}$ & $9.3183 e-02$ & $7.0120 \mathrm{e}-02$ & $4.4773 e-02$ & $2.0546 \mathrm{e}-02$ & $1.0545 \mathrm{e}-02$ \\
\hline $2^{-12}$ & $1.7017 \mathrm{e}-01$ & $1.0083 e-01$ & $6.2216 \mathrm{e}-02$ & $3.9526 \mathrm{e}-02$ & $2.0493 e-02$ \\
\hline $2^{-18}$ & $2.5614 \mathrm{e}-01$ & $2.1031 \mathrm{e}-01$ & $1.3406 \mathrm{e}-01$ & $8.5618 e-02$ & $4.8834 \mathrm{e}-02$ \\
\hline
\end{tabular}

\section{Conclusion}

The accelerated nonstandard finite difference method is presented for solving the singularly perturbed BurgerHuxley equation. The nonlinear terms are linearized by the quasilinearization technique. First-order finite difference approximation for the discretization of a time derivative and the nonstandard methodology of Micken's procedure is applied for the spatial derivatives. To accelerate the convergence of the method, the Richardson extrapolation technique is applied. It is provided that from numerical results, the method gives a better accurate solution with a higher order of convergence than some existing methods in the literature. Therefore, the presented method is second-order convergent and gives an accurate solution for solving the singularly perturbed Burger-Huxley equation.

Table 2 Comparison of maximum absolute errors for Example 2

\begin{tabular}{|c|c|c|c|c|c|}
\hline$\varepsilon \downarrow M / N \rightarrow$ & $32 / 20$ & $64 / 40$ & $128 / 80$ & $256 / 160$ & $512 / 320$ \\
\hline \multicolumn{6}{|c|}{ After extrapolation } \\
\hline $2^{-6}$ & $1.0956 \mathrm{e}-04$ & $2.8034 \mathrm{e}-05$ & $7.6248 \mathrm{e}-06$ & $4.1959 \mathrm{e}-06$ & $2.2851 e-06$ \\
\hline $2^{-8}$ & $1.5301 \mathrm{e}-04$ & $4.9218 \mathrm{e}-05$ & $1.3827 \mathrm{e}-05$ & $3.6543 e-06$ & $9.3878 \mathrm{e}-07$ \\
\hline $2^{-10}$ & $2.0315 \mathrm{e}-04$ & $9.4930 \mathrm{e}-05$ & $3.6697 \mathrm{e}-05$ & $1.1291 \mathrm{e}-05$ & $3.0711 \mathrm{e}-06$ \\
\hline $2^{-12}$ & $1.9354 \mathrm{e}-04$ & $8.7202 e-05$ & $5.8753 e-05$ & $3.1369 e-05$ & $1.1375 e-05$ \\
\hline $2^{-18}$ & $2.7286 e-04$ & $7.2496 \mathrm{e}-05$ & $1.8396 \mathrm{e}-05$ & $4.4949 \mathrm{e}-06$ & $2.6628 \mathrm{e}-06$ \\
\hline \multicolumn{6}{|l|}{ Results in [6] } \\
\hline $2^{-6}$ & $3.8767 \mathrm{e}-02$ & $1.8983 e-02$ & $9.6122 \mathrm{e}-03$ & $5.0867 e-03$ & $2.6211 \mathrm{e}-03$ \\
\hline $2^{-8}$ & $4.4450 \mathrm{e}-02$ & $2.0109 \mathrm{e}-02$ & $1.0519 \mathrm{e}-02$ & $5.9653 \mathrm{e}-02$ & $3.1896 \mathrm{e}-03$ \\
\hline $2^{-10}$ & $8.3339 e-02$ & $6.6120 \mathrm{e}-02$ & $4.0769 e-02$ & $1.9284 \mathrm{e}-02$ & $9.1775 e-03$ \\
\hline $2^{-12}$ & $1.8762 \mathrm{e}-01$ & $8.4106 \mathrm{e}-02$ & $5.7234 \mathrm{e}-02$ & $3.309 e-02$ & $1.9041 \mathrm{e}-02$ \\
\hline $2^{-18}$ & $2.8299 \mathrm{e}-01$ & $1.7036 \mathrm{e}-01$ & $1.1805 \mathrm{e}-01$ & $7.4251 \mathrm{e}-02$ & $4.1879 e-02$ \\
\hline
\end{tabular}


Table 3 Comparison of rate of convergence for Example 1

\begin{tabular}{lllll}
\hline $\boldsymbol{\varepsilon} \downarrow \mathbf{M} / \mathbf{N} \rightarrow$ & $\mathbf{3 2 / 2 0}$ & $\mathbf{6 4 / 4 0}$ & $\mathbf{1 2 8 / 8 0}$ & $\mathbf{2 5 6 / 1 6 0}$ \\
\hline After extrapolation & & & & \\
$2^{-6}$ & 1.9098 & 1.9894 & 1.7283 & 1.6506 \\
$2^{-8}$ & 1.8727 & 1.9458 & 1.9784 & 1.9936 \\
$2^{-10}$ & 1.8710 & 1.9392 & 1.9706 & 1.9868 \\
$2^{-12}$ & 1.8715 & 1.9350 & 1.9696 & 1.9852 \\
$2^{-18}$ & 1.8713 & 1.9339 & 1.9692 & 1.9845 \\
Before extrapolation & & & & \\
$2^{-6}$ & 0.8839 & 0.9566 & 0.9989 & 1.0228 \\
$2^{-8}$ & 0.8839 & 0.9566 & 0.9989 & 1.0228 \\
$2^{-10}$ & 0.8769 & 0.9385 & 0.9695 & 0.9851 \\
$2^{-12}$ & 0.8778 & 0.9388 & 0.9686 & 0.9846 \\
$2^{-18}$ & 0.8782 & 0.9390 & 0.9679 & 0.9845 \\
Results in [6] & & & & \\
$2^{-6}$ & 0.5170 & 0.9724 & 0.8966 & 0.9504 \\
$2^{-8}$ & 0.9856 & 0.7971 & 0.8362 & 0.9119 \\
$2^{-10}$ & 0.4102 & 0.6472 & 1.1238 & 0.9623 \\
$2^{-12}$ & 0.7550 & 0.6966 & 0.6544 & 0.9477 \\
$2^{-18}$ & 0.2844 & 0.6496 & 0.6469 & 0.8100 \\
\hline & & & & \\
\hline
\end{tabular}

\section{Limitations}

During the quasi-linearization process we used only the first iteration. If more number of iterations were done, the proposed scheme can have more accurate solution than the existing results. Further, the scheme can more illustrate the physical behaviour of the problem under consideration.

\section{Abbreviations}

ODE: Ordinary differential equation; TE: Truncation errors.

\section{Acknowledgements}

The authors thank Jimma University for their material supports.

\section{Authors' contributions}

MJK carried out scheme development, MATLAB coding and numerical experimentation. GFD formulated the problem, design, and draft the manuscript. Both authors read and approved the final manuscript.

\section{Funding}

No funding organization for this research work.

\section{Availability of data and materials}

No additional data is used for this research work.

\section{Declarations}

Ethics approval and consent to participate Not applicable.

\section{Consent for publication}

Not applicable.

\section{Competing interests}

The authors declare that they have no competing interests.

Received: 18 September 2021 Accepted: 23 November 2021

Published online: 11 December 2021

References

1. Bullo T, Duressa GF, Degla G. Accelerated fitted operator finite difference method for singularly perturbed parabolic reaction-diffusion problems. Computat Methods Diff Eq. 2021;9(3):886-98.

2. Bullo TA, Degla GA, Duressa GF. Uniformly convergent higher-order finite difference scheme for singularly perturbed parabolic problems with nonsmooth data. J Appl Math Comput Mech. 2021:20(1):5-16.

3. Mekonnen TB, Duressa GF. A computational method for singularly perturbed two-parameter parabolic convection-diffusion problems. Cogent Math Stat. 2020;7(1):1829277.

4. Bullo TA, Duressa GF, Degla GA. Robust finite difference method for singularly perturbed two-parameter parabolic convection-diffusion problems. Int J Comput Methods. 2021;18(2):2050034.

5. Woldaregay MM, Duressa GF. A uniformly convergent numerical method for singularly perturbed delay parabolic differential equations arising in computational neuroscience. Kragujevac J Math. 2022:46(1):65-84.

6. Liu LB, Liang Y, Zhang J, Bao X. A robust adaptive grid method for singularly perturbed Burger-Huxley equations. Elect Res Arch. 2020:28(4):1439.

7. Mohanty RK, Dai W, Liu D. Operator compact method of accuracy two in time and four in space for the solution of time-dependent BurgersHuxley equation. Numerical Algorithm. 2015;70(3):591-605.

8. Mohammadi R. B-spline collocation algorithm for the numerical solution of the generalized Burger's-Huxley equation. Numer Methods Partial Diff Equ. 2013;29(4):1173-91.

9. Khattak AJ. A computational meshless method for the generalized Burger's-Huxley equation. Appl Math Model. 2009;33(9):3718-29.

10. Mittal RC, Tripathi A. Numerical solutions of generalized Burgers-Fisher and generalized Burgers-Huxley equations using collocation of cubic B-splines. Int J Comput Math. 2015;92(5):1053-77.

11. Kaushik A, Sharma MD. A uniformly convergent numerical method on non-uniform mesh for singularly perturbed unsteady Burger-Huxley equation. Appl Math Comput. 2008;195(2):688-706.

12. Mickens RE. Advances in the applications of nonstandard finite difference schemes. World Scientific; 2005.

13. Jima KM, File DG. Implicit Finite Difference Scheme for Singularly Perturbed Burger-Huxley Equations. 2022;35(1):87-100.

14. Kabeto MJ, Duressa GF. Robust numerical method for singularly perturbed semilinear parabolic differential difference equations. Math Comput Simul. 2021;188:537-47.

\section{Publisher's Note}

Springer Nature remains neutral with regard to jurisdictional claims in published maps and institutional affiliations.

Ready to submit your research? Choose BMC and benefit from:

- fast, convenient online submission

- thorough peer review by experienced researchers in your field

- rapid publication on acceptance

- support for research data, including large and complex data types

- gold Open Access which fosters wider collaboration and increased citations

- maximum visibility for your research: over 100M website views per year

At $\mathrm{BMC}$, research is always in progress.

Learn more biomedcentral.com/submissions 